\title{
The existence of sign-changing solution for a class of quasilinear Schrödinger-Poisson systems via perturbation method
}

\author{
Lizhen Chen ${ }^{1}$, Xiaojing Feng ${ }^{2 *}$ and Xinan $\mathrm{HaO}^{3}$
}

\section{"Correspondence: \\ fengxj@sxu.edu.cn \\ ${ }^{2}$ School of Mathematical Sciences, \\ Shanxi University, Taiyuan, People's Republic of China \\ Full list of author information is \\ available at the end of the article}

\begin{abstract}
This paper is concerned with the existence of a sign-changing solution to a class of quasilinear Schrödinger-Poisson systems. There are some technical difficulties in applying variational methods directly to the problem because the quasilinear term makes it impossible to find a suitable space in which the corresponding functional possesses both smoothness and compactness properties. In order to overcome the difficulties caused by nonlocal term and quasi-linear term, we shall apply the perturbation method by adding a 4-Laplacian operator to consider the perturbation problem. And then, by using the approximation technique, a sign-changing solution with precisely two nodal domains is derived.
\end{abstract}

MSC: $35 J 20 ; 35 J 60$

Keywords: Quasilinear Schrödinger-Poisson system; Perturbation method; Sign-changing solution

\section{Introduction and main results}

In this paper, we consider the existence of a sign-changing solution for the following system:

$$
\begin{cases}-\Delta u+V(x) u+\phi u-\frac{1}{2} u \Delta u^{2}=f(u), & \text { in } \mathbb{R}^{3}, \\ -\Delta \phi=u^{2}, & \text { in } \mathbb{R}^{3}\end{cases}
$$

where $V$ is a continuous potential function and $f$ is an appropriate nonlinear function. On the potential $V$, we make the following assumption:

(V) $V \in C\left(\mathbb{R}^{3}, \mathbb{R}\right), \lim _{|x| \rightarrow \infty} V(x)=\infty$, and $V(x) \geq m>0$ for some constant $m$.

According to a classical model, the interaction of a charge particle with an electromagnetic field can be described by coupling the nonlinear Schrödinger equations and Poisson equations. In the recent years, there has been a lot of work dealing with the following Schrödinger-Poisson systems:

$$
\begin{cases}-\Delta u+V(x) u+\phi u=f(x, u), & \text { in } \mathbb{R}^{3}, \\ -\Delta \phi=u^{2}, & \text { in } \mathbb{R}^{3} .\end{cases}
$$

(c) The Author(s) 2019. This article is distributed under the terms of the Creative Commons Attribution 4.0 International License (http://creativecommons.org/licenses/by/4.0/), which permits unrestricted use, distribution, and reproduction in any medium, provided you give appropriate credit to the original author(s) and the source, provide a link to the Creative Commons license, and indicate if changes were made. 
For example, Ambrosetti and Ruiz in [4] obtained the multiplicity results of (1.2) by using the variational methods and Pohožaev equality. By using fountain theorem for the subcritical case and the symmetric mountain pass theorem, Wang, Radulescu, and Zhang [37] studied the existence of infinitely many solutions for a class of fractional KirchhoffSchrödinger-Poisson system under certain assumptions. Goubet and Hamraoui in [19] investigated both numerically and theoretically the influence of a defect on the blow-up of radial solutions to a cubic nonlinear Schrödinger equation in dimension 2. In [36], Trabelsi showed the global well-posedness of a higher-order nonlinear Schrödinger equation. Specifically, the author considered a system of infinitely many coupled higher-order Schrödinger-Poisson-Slater equations with a self-consistent Coulomb potential. By applying non-Nehari manifold method, Wen and Chen [40] established the existence of the Nehari-type ground state solutions for asymptotically periodic Schrödinger-Poisson systems involving Hartree-type nonlinearities. Wang and Zhou [38] considered the existence and nonexistence of solution to (1.2) under the assumption that $f$ is asymptotically linear at infinity. By using the constraint variational method and the Brouwer degree theory, Wang and Zhou [39] proved that system (1.2) has a sign-changing solution under suitable assumptions. Zhao and Zhao in [46] studied the existence and multiplicity of solutions to (1.2) with $f(x, u)=|u|^{p-1} u, 2<p \leq 3$ via variational methods. When $V$ is periodic or asymptotically periodic and $f$ does not satisfy the Ambrosetti-Rabinowitz condition, Alves, Souto, and Soares [2] established the existence of positive ground state solutions by using the mountain pass theorem. Zhao, Liu, and Zhao in [45] considered the existence of nontrivial solution and concentration results for a class of Schrödinger-Poisson equations via variational methods. Assumption $(V)$ was originally used by Rabinowitz in [30] for a semi-linear problem and also used by Omana and Willem in [28] for Hamiltonian systems and by Costa in [12] for elliptic systems. In particular, Bartsch and Wang [7] introduced the assumption:

$\left(V^{\prime}\right) V \in C\left(\mathbb{R}^{3}, \mathbb{R}\right)$ and $\inf _{x \in \mathbb{R}^{3}} V(x) \geq \beta>0$. Moreover, for every $M>0$, meas $\left\{x \in \mathbb{R}^{3}:\right.$ $V(x) \leq M\}<\infty$, where meas(.) denotes the Lebesgue measure in $\mathbb{R}^{3}$,

which is also used to overcome the lack of compactness. It implies the coercive condition $V(x) \rightarrow \infty$ as $|x| \rightarrow \infty$. Hence, assumption $(V)$ can be replaced by $\left(V^{\prime}\right)$.

There are also many researchers dealing with the following quasilinear Schrödinger equations:

$$
-\Delta u+V(x) u-\kappa \Delta\left(l\left(u^{2}\right)\right) l^{\prime}\left(u^{2}\right) u=f(x, u), \quad x \in \mathbb{R}^{3},
$$

where $V$ is a proper potential function, $\kappa$ is a real constant, and $f, l$ are real functions. Solutions of (1.3) are related to the standing wave solutions for quasilinear Schrödinger equations of the form

$$
i \psi_{t}+\Delta \psi-V(x) \psi+\kappa \Delta\left(l\left(|\psi|^{2}\right)\right) l^{\prime}\left(|\psi|^{2}\right)+f(x, \psi)=0, \quad x \in \mathbb{R}^{3} .
$$

There are many different forms about (1.3) with different expressions for $l$. When $l$ is a constant, it converts to a semilinear problem. When $l(s)=s^{\alpha}, \alpha \geq 1$, and $l(s)=(1+s)^{1 / 2}$, it changes into some special quasilinear problems. Moreover, it turns into a general quasilinear problem when $l$ is a general function. 
In particular, when $l(s)=s,(1.3)$ becomes quasilinear Schrödinger equation as follows:

$$
\left.-\Delta u+V(x) u-\kappa \Delta\left(|u|^{2}\right)\right) u=f(x, u), \quad x \in \mathbb{R}^{3},
$$

which has been studied extensively in recent years. The influence of the signs of parameter $\kappa$ on the existence of solutions is important. For instance, Tang, Zhang, and Zhang in [44] obtained the existence of infinitely many nontrivial solutions of (1.4) with $\kappa=1$ by using the dual approach and mountain pass theorem when the nonlinearity $f(x, u)$ is superlinear growth. By virtue of mountain pass theorem and Moser iteration method, Alves, Wang, and Shen in [3] used another change of variable to deal with the nontrivial solution for (1.4) when $\kappa<0$.

As is known to all, there are some technical difficulties in applying variational methods directly to the quasilinear equations because the quasilinear term makes it impossible to find a suitable space in which the corresponding functional possesses both smoothness and compactness properties. In order to overcome these difficulties, there are three methods which were used before. The first one is the constrained minimization or the Nehari method. By using this method, Poppenberg, Schmitt, and Wang in [29] obtained the existence of standing wave solutions for a class of quasilinear Schrödinger equations with strongly singular nonlinearities; Ruiz and Siciliano in [32] derived the existence of ground states; Liu, Wang, and Wang in [23] established the positive solutions and signchanging solutions; and Liu, Wang, and Wang in [22] considered the existence of ground states of soliton-type solutions. The second method is change of variables (see $[9,11,13-$ $15,21,22,42])$. In [11] Colin and Jeanjean obtained the existence of nontrivial solution to (1.5) under the nonautonomous cases and autonomous cases. Deng, Peng, and Wang in [13] obtained a sign-changing minimizer of (1.4) by adopting the minimization argument. Chen et al. in [9] proved the existence of sign-changing solutions with two nodal domains for (1.4) with a Kirchhoff-type perturbation by using Miranda's theorem and deformation lemma. Deng, Peng, and Yan in $[14,15]$ investigated a generalized quasilinear Schrödinger equation with critical exponents by using a change of variables and variational argument. $\mathrm{Wu}$ and $\mathrm{Wu}$ in [42] considered the existence of radial solutions for a class of quasilinear Schrödinger equations by using the variational argument and the Pohozaev-type identity. The last one is the perturbation method which was introduced by Liu, Liu, and Wang in [25]. Some existence results for positive solutions, negative solutions, and a sequence of high energy solutions were obtained in [43] by using the variational method. Wu and Wu in [41] studied a class of Schrödinger-Kirchhoff quasilinear problems and proved the existence of infinitely many small energy solutions by applying Clark's theorem to a perturbation functional. Jeanjean, Luo, and Wang in [20] considered the existence of two normalized solutions by relying on the perturbation method.

When $l$ is a constant, (1.3) can be written into the following form:

$$
-\Delta u+V(x) u=f(x, u), \quad x \in \mathbb{R}^{3} .
$$

There are different ways to get the sign-changing solutions of equation (1.5). By using the variational argument and a version of deformation lemma, Castro, Cossio, and Neuberger [8] proved that (1.5) has at least three nontrivial solutions. Noussair and Wei in [27] established the existence of nodal solutions in a bounded domain based on Ekeland's 
variational principle and implicit function theorem. Bartsch, Liu, and Weth in [6] came up with infinitely many nodal solutions via construct invariant sets and descending flow. Bartsch, Liu, and Weth in [5] proved the existence of sign-changing solutions of (1.5) with superlinear and subcritical nonlinearity term by combining variational method with the Brouwer degree theory.

It is worth emphasizing that these methods in finding sign-changing solutions rely on the following decomposition. For $u \in H^{1}\left(\mathbb{R}^{3}\right)$,

$$
\begin{aligned}
& \left\langle I_{0}^{\prime}(u), u^{+}\right\rangle=\left\langle I_{0}^{\prime}\left(u^{+}\right), u^{+}\right\rangle, \quad\left\langle I_{0}^{\prime}(u), u^{-}\right\rangle=\left\langle I_{0}^{\prime}\left(u^{-}\right), u^{-}\right\rangle, \\
& I_{0}(u)=I_{0}\left(u^{+}\right)+I_{0}\left(u^{-}\right),
\end{aligned}
$$

where $I_{0}$ is the energy functional associated to (1.5), and

$$
u^{+}(x)=\max \{u(x), 0\}, \quad u^{-}(x)=\min \{u(x), 0\} .
$$

But, for the functional $I$ corresponding to (1.2), we deduce by the nonlocal term $\phi_{u}(x)=$ $\phi_{u^{+}}(x)+\phi_{u^{-}}$that

$$
I(u)=I\left(u^{+}\right)+I\left(u^{-}\right)+\frac{1}{4} \int_{\mathbb{R}^{3}} \phi_{u^{-}}\left(u^{+}\right)^{2} d x+\frac{1}{4} \int_{\mathbb{R}^{3}} \phi_{u^{+}}\left(u^{-}\right)^{2} d x,
$$

and

$$
\begin{aligned}
& \left\langle I^{\prime}(u), u^{+}\right\rangle=\left\langle I^{\prime}\left(u^{+}\right), u^{+}\right\rangle+\int_{\mathbb{R}^{3}} \phi_{u^{-}}\left(u^{+}\right)^{2} d x, \\
& \left\langle I^{\prime}(u), u^{-}\right\rangle=\left\langle I^{\prime}\left(u^{-}\right), u^{-}\right\rangle+\int_{\mathbb{R}^{3}} \phi_{u^{+}}\left(u^{-}\right)^{2} d x .
\end{aligned}
$$

It is clear that the functional $I$ does not satisfy decomposition (1.6) and (1.7) any more. Hence the methods of obtaining sign-changing solutions of (1.5) cannot be applied to system (1.1).

In fact, there are some essential differences in investigating the sign-changing solutions between local and nonlocal equations. In particular, Wang and Zhou in [39] obtained a sign-changing solution for system (1.2) by seeking minimizer of the energy functional $I$ over the following constraint:

$$
\mathcal{M}_{0}=\left\{u \in H^{1}\left(\mathbb{R}^{3}\right): u^{ \pm} \neq 0,\left\langle I^{\prime}(u), u^{+}\right\rangle=\left\langle I^{\prime}(u), u^{-}\right\rangle=0\right\} .
$$

This argument mainly shows that there is a minimizer of $I$ constrained on $\mathcal{M}_{0}$ and then verifies that the minimizer is a critical point of $I$ via quantitative deformation lemma and degree theory. By using the method, the sign-changing solution for some nonlocal equations is constructed (see $[1,9,10,17,18,21,33-35]$ ). The Choquard equation was studied by Ghimenti and Schaftingen in [18]. The nonlinear Schrödinger-Poisson systems in bounded domains were considered by Alves and Souto in [1]. The Schrödinger-Poisson type problems in $\mathbb{R}^{3}$ were also researched by Chen and Tang in [10] and Shuai and Wang in [34]. The Kirchhoff equation was investigated by Figueiredo and Nascimento in [17], Tang and Chen in [35], and Shuai in [33]. The quasilinear Schrödinger equations with a 
Kirchhoff-type perturbation were discussed by Li, Zhu, and Liang in [21] and Chen et al. in [9].

Motivated by the above papers, we consider the existence of the sign-changing solution to system (1.1). Let $H_{V}^{1}\left(\mathbb{R}^{3}\right):=\left\{u \in H^{1}\left(\mathbb{R}^{3}\right) \mid \int_{\mathbb{R}^{3}} V(x) u^{2} d x<+\infty\right\}$. For system (1.1), we want to look for $u \in H_{V}^{1} \cap L^{\infty}\left(\mathbb{R}^{3}\right)$ such that for all $\varphi \in C^{\infty}\left(\mathbb{R}^{3}\right)$ satisfying

$$
\begin{aligned}
& \int_{\mathbb{R}^{3}}(\nabla u \nabla \varphi+V(x) u \varphi) d x+\int_{\mathbb{R}^{3}} u^{2} \nabla u \nabla \varphi d x+\int_{\mathbb{R}^{3}}|\nabla u|^{2} u \varphi d x \\
& +\int_{\mathbb{R}^{3}} \phi_{u} u \varphi d x-\int_{\mathbb{R}^{3}} f(u) \varphi d x=0,
\end{aligned}
$$

which is formally associated to the energy functional given by

$$
\begin{aligned}
J(u)= & \frac{1}{2} \int_{\mathbb{R}^{3}}\left(|\nabla u|^{2}+V(x) u^{2}+u^{2}|\nabla u|^{2}\right) d x+\frac{1}{4} \int_{\mathbb{R}^{3}} \phi_{u} u^{2} d x \\
& -\int_{\mathbb{R}^{3}} F(u) d x, \quad u \in H_{V}^{1} \cap L^{\infty}\left(\mathbb{R}^{3}\right),
\end{aligned}
$$

where $F(u)=\int_{0}^{u} f(s) d s$ and $\phi_{u}$ is given in (2.1). We cannot apply variational methods directly to the problem because the quasilinear term makes it impossible to find a suitable space in which the corresponding functional possesses both smoothness and compactness properties. On the other hand, it is difficult to use the dual approach to consider the sign-changing solution of (1.1) because of the nonlocal term. Thereby, we would employ the method in [25] and [24]. In fact, we use the approximation's method by adding a 4Laplacian operator and firstly consider the sign-changing critical point of the perturbed functional:

$$
J_{\lambda}(u)=J(u)+\frac{\lambda}{4} \int_{\mathbb{R}^{3}}\left(|\nabla u|^{4}+u^{4}\right) d x,
$$

where $\lambda \in(0,1]$. Then, by using the approximation technique and Moser's iteration method, the existence of sign-changing solution to system (1.1) is derived. Our result reads as follows.

Theorem 1.1 Assume that $(V)$ and the following conditions hold:

$\left(f_{1}\right) f \in C^{1}(\mathbb{R}, \mathbb{R})$

$\left(f_{2}\right) \lim _{s \rightarrow 0} \frac{f(s)}{s}=\lim _{|s| \rightarrow \infty} \frac{f(s)}{s^{11}}=0$;

$\left(f_{3}\right)$ there exists $\mu>4$ such that

$$
0<\mu F(s)=\mu \int_{0}^{s} f(t) d t \leq s f(s), \quad s \in \mathbb{R} \backslash\{0\} ;
$$

$\left(f_{4}\right) \frac{f(t)}{|t|^{3}}$ is increasing on $(-\infty, 0)$ and $(0, \infty)$, respectively.

Then problem (1.1) possesses at least a sign-changing solution which has precisely two nodal domains.

Remark 1.2 Throughout the paper, we denote by $C>0$ various positive constants which may vary from line to line and are not essential to the problem. 
The paper is organized as follows: in Sect. 2, some preliminary results are presented. Section 3 is devoted to the proof of Theorem 1.1.

\section{Preliminary}

In this section, we give some notations which will be used throughout this paper. Let $L^{p}\left(\mathbb{R}^{3}\right)$ be the usual Lebesgue space with the norm $\|u\|_{p}=\left(\int_{\mathbb{R}^{3}}|u|^{p} d x\right)^{1 / p}$ and $H^{1}\left(\mathbb{R}^{3}\right)$ be the completion of $C_{0}^{\infty}\left(\mathbb{R}^{3}\right)$ with respect to the norm

$$
\|u\|_{H}^{2}=\int_{\mathbb{R}^{3}}\left(|\nabla u|^{2}+u^{2}\right) d x
$$

Moreover, we denote the completion of $C_{0}^{\infty}\left(\mathbb{R}^{3}\right)$ with respect to the norm

$$
\|u\|_{D^{1,2}}^{2}=\int_{\mathbb{R}^{3}}|\nabla u|^{2} d x
$$

by $D^{1,2}=D^{1,2}\left(\mathbb{R}^{3}\right)$. In order to deal with the perturbation functional $J_{\lambda}$, we need the space

$$
X=W^{1,4}\left(\mathbb{R}^{3}\right) \cap H_{V}^{1}\left(\mathbb{R}^{3}\right),
$$

where

$$
H_{V}^{1}\left(\mathbb{R}^{3}\right):=\left\{u \in H^{1}\left(\mathbb{R}^{3}\right) \mid \int_{\mathbb{R}^{3}} V(x) u^{2} d x<+\infty\right\}
$$

which is a Hilbert space endowed with the norm

$$
\|u\|_{H_{V}^{1}}=\left(\int_{\mathbb{R}^{3}}\left(|\nabla u|^{2}+V(x) u^{2}\right) d x\right)^{1 / 2},
$$

and $W^{1,4}\left(\mathbb{R}^{3}\right)$ endowed with the norm

$$
\|u\|_{W}=\left(\int_{\mathbb{R}^{3}}\left(|\nabla u|^{4}+u^{4}\right) d x\right)^{1 / 4} .
$$

The norm of $X$ is denoted by

$$
\|u\|=\left(\|u\|_{W}^{2}+\|u\|_{H_{V}^{1}}^{2}\right)^{1 / 2} .
$$

From $\left(f_{1}\right)$ and $\left(f_{2}\right)$, it is normal to verify that $J_{\lambda} \in C^{1}(X, \mathbb{R})$ for all $\varphi \in X$, and

$$
\begin{aligned}
\left\langle J_{\lambda}^{\prime}(u), \varphi\right\rangle= & \lambda \int_{\mathbb{R}^{3}}\left(|\nabla u|^{2} \nabla u \nabla \varphi+u^{3} \varphi\right) d x+\int_{\mathbb{R}^{3}}(\nabla u \nabla \varphi+V(x) u \varphi) d x+\int_{\mathbb{R}^{3}} \phi_{u} u \varphi d x \\
& +\int_{\mathbb{R}^{3}}|\nabla u|^{2} u \varphi d x+\int_{\mathbb{R}^{3}} u^{2} \nabla u \nabla \varphi d x-\int_{\mathbb{R}^{3}} f(u) \varphi d x .
\end{aligned}
$$

In the proof of Theorem 1.1, we first prove that, for fixing $\lambda \in(0,1]$, the nodal set

$$
\mathcal{M}_{\lambda}=\left\{u \in X: u^{ \pm} \neq 0,\left\langle J_{\lambda}^{\prime}(u), u^{+}\right\rangle=\left\langle J_{\lambda}^{\prime}(u), u^{-}\right\rangle=0\right\}
$$


is nonempty and

$$
m_{\lambda}=\inf _{u \in \mathcal{M}_{\lambda}} J_{\lambda}(u)>0
$$

Then, we show that there is $u_{\lambda} \in \mathcal{M}_{\lambda}$ such that

$$
J_{\lambda}\left(u_{\lambda}\right)=\inf _{u \in \mathcal{M}_{\lambda}} J_{\lambda}(u)
$$

Furthermore, we prove that $u_{\lambda}$ is a critical point of $J_{\lambda}$ via quantitative deformation lemma and degree theory. Finally, we obtain the convergence property of $u_{\lambda}$ as $\lambda \rightarrow 0$, and thus the sign-changing solution of (1.1) is derived.

We observe that by the Lax-Milgram theorem, for given $u \in H^{1}\left(\mathbb{R}^{3}\right)$, there exists a unique solution $\phi=\phi_{u} \in D^{1,2}$ satisfying $-\Delta \phi_{u}=u^{2}$ in a weak sense. The function $\phi_{u}$ is represented by

$$
\phi_{u}(x)=\frac{1}{4 \pi} \int_{\mathbb{R}^{3}} \frac{u^{2}(y)}{|x-y|} d y
$$

and it has the following properties.

Lemma 2.1 ([31]) The following properties hold:

(i) There exists $C>0$ such that, for any $u \in H^{1}\left(\mathbb{R}^{3}\right)$,

$$
\left\|\phi_{u}\right\|_{D^{1,2}} \leq C\|u\|_{12 / 5}^{2}, \quad \int_{\mathbb{R}^{3}}\left|\nabla \phi_{u}\right|^{2} d x=\int_{\mathbb{R}^{3}} \phi_{u} u^{2} d x \leq C\|u\|_{H}^{4}
$$

(ii) $\phi_{u} \geq 0$ for all $u \in H^{1}\left(\mathbb{R}^{3}\right)$;

(iii) If $u$ is radially symmetric, then $\phi_{u}$ is radial;

(iv) $\phi_{t u}=t^{2} \phi_{u}$ for all $t>0$ and $u \in H^{1}\left(\mathbb{R}^{3}\right)$;

(v) If $u_{j} \rightarrow u$ weakly in $H_{V}^{1}\left(\mathbb{R}^{3}\right)$, then, up to a subsequence, $\phi_{u_{j}} \rightarrow \phi_{u}$ in $D^{1,2}$ and

$$
\int_{\mathbb{R}^{3}} \phi_{u_{j}} u_{j}^{2} d x \rightarrow \int_{\mathbb{R}^{3}} \phi_{u} u^{2} d x
$$

\section{Proofs of the main result}

In this section, we first apply the methods of [10] to show the following lemma which will play the fundamental role in our proof.

Lemma 3.1 Assume that $(V),\left(f_{1}\right),\left(f_{2}\right)$, and $\left(f_{4}\right)$ hold. Then, for any $u=u^{+}+u^{-} \in X$, there holds

$$
\begin{aligned}
J_{\lambda}(u) & -J_{\lambda}\left(s u^{+}+t u^{-}\right) \\
\geq & \frac{1-s^{4}}{4}\left\langle J_{\lambda}^{\prime}(u), u^{+}\right\rangle+\frac{1-t^{4}}{4}\left\langle J_{\lambda}^{\prime}(u), u^{-}\right\rangle \\
& +\frac{\left(1-s^{2}\right)^{2}}{4}\left\|u^{+}\right\|_{H_{V}^{1}}^{2}+\frac{\left(1-t^{2}\right)^{2}}{4}\left\|u^{-}\right\|_{H_{V}^{1}}^{2}+\frac{\left(s^{2}-t^{2}\right)^{2}}{4} \int_{\mathbb{R}^{3}} \phi_{u^{+}}\left(u^{-}\right)^{2} d x, \quad s, t \geq 0 .
\end{aligned}
$$


Proof We deduce from $\left(f_{4}\right)$ that, for any $t \geq 0, \tau \in \mathbb{R}$,

$$
\frac{1-t^{4}}{4} \tau f(\tau)+F(t \tau)-F(\tau)=\int_{t}^{1}\left(\frac{f(\tau)}{\tau^{3}}-\frac{f(s \tau)}{(s \tau)^{3}}\right) s^{3} \tau^{4} d s \geq 0
$$

Thus, for any $t, s \geq 0$, it follows

$$
\begin{aligned}
& J_{\lambda}(u)-J_{\lambda}\left(s u^{+}+t u^{-}\right) \\
& =\frac{\lambda}{4}\left(\|u\|_{W}^{4}-\left\|s u^{+}+t u^{-}\right\|_{W}^{4}\right)+\frac{1}{4} \int_{\mathbb{R}^{3}}\left[\phi_{u} u^{2}-\phi_{s u^{+}+t u^{-}}\left(s u^{+}+t u^{-}\right)^{2}\right] d x \\
& +\frac{1}{2}\left(\|u\|_{H_{V}^{1}}^{2}-\left\|s u^{+}+t u^{-}\right\|_{H_{V}^{1}}^{2}\right) \\
& +\frac{1}{2} \int_{\mathbb{R}^{3}}\left[u^{2}|\nabla u|^{2}-\left(s u^{+}+t u^{-}\right)^{2}\left|\nabla\left(s u^{+}+t u^{-}\right)\right|^{2}\right] d x \\
& +\int_{\mathbb{R}^{3}}\left[F\left(s u^{+}+t u^{-}\right)-F(u)\right] d x \\
& =\frac{\lambda}{4}\left(\left\|u^{+}\right\|_{W}^{4}+\left\|u^{-}\right\|_{W}^{4}-s^{4}\left\|u^{+}\right\|_{W}^{4}-t^{4}\left\|u^{-}\right\|_{W}^{4}\right) \\
& +\frac{1}{4} \int_{\mathbb{R}^{3}}\left[\phi_{u^{+}}\left(u^{+}\right)^{2}+\phi_{u^{-}}\left(u^{-}\right)^{2}+2 \phi_{u^{+}}\left(u^{-}\right)^{2}-s^{4} \phi_{u^{+}}\left(u^{+}\right)^{2}\right. \\
& \left.-t^{4} \phi_{u^{-}}\left(u^{-}\right)^{2}-2 s^{2} t^{2} \phi_{u^{+}}\left(u^{-}\right)^{2}\right] d x \\
& +\frac{1}{2}\left(\left\|u^{+}\right\|_{H_{V}^{1}}^{2}+\left\|u^{-}\right\|_{H_{V}^{1}}^{2}-s^{2}\left\|u^{+}\right\|_{H_{V}^{1}}^{2}-t^{2}\left\|u^{-}\right\|_{H_{V}^{1}}^{2}\right) \\
& +\frac{1}{2} \int_{\mathbb{R}^{3}}\left[\left(u^{+}\right)^{2}\left|\nabla u^{+}\right|^{2}+\left(u^{-}\right)^{2}\left|\nabla u^{-}\right|^{2}-s^{4}\left(u^{+}\right)^{2}\left|\nabla u^{+}\right|^{2}-t^{4}\left(u^{-}\right)^{2}\left|\nabla u^{-}\right|^{2}\right] d x \\
& +\int_{\mathbb{R}^{3}}\left[F\left(s u^{+}\right)+F\left(t u^{-}\right)-F\left(u^{+}\right)-F\left(u^{-}\right)\right] d x \\
& =\frac{1-s^{4}}{4}\left\langle J_{\lambda}^{\prime}(u), u^{+}\right\rangle+\frac{1-t^{4}}{4}\left\langle J_{\lambda}^{\prime}(u), u^{-}\right\rangle \\
& +\frac{\left(1-s^{2}\right)^{2}}{4}\left\|u^{+}\right\|_{H_{V}^{1}}^{2}+\frac{\left(1-t^{2}\right)^{2}}{4}\left\|u^{-}\right\|_{H_{V}^{1}}^{2}+\frac{\left(s^{2}-t^{2}\right)^{2}}{4} \int_{\mathbb{R}^{3}} \phi_{u^{+}}\left(u^{-}\right)^{2} d x \\
& +\int_{\mathbb{R}^{3}}\left[\frac{1-s^{4}}{4} f\left(u^{+}\right) u^{+}+F\left(s u^{+}\right)-F\left(u^{+}\right)\right] d x \\
& +\int_{\mathbb{R}^{3}}\left[\frac{1-t^{4}}{4} f\left(u^{-}\right) u^{-}+F\left(t u^{-}\right)-F\left(u^{-}\right)\right] d x \\
& \geq \frac{1-s^{4}}{4}\left\langle J_{\lambda}^{\prime}(u), u^{+}\right\rangle+\frac{1-t^{4}}{4}\left\langle J_{\lambda}^{\prime}(u), u^{-}\right\rangle \\
& +\frac{\left(1-s^{2}\right)^{2}}{4}\left\|u^{+}\right\|_{H_{V}^{1}}^{2}+\frac{\left(1-t^{2}\right)^{2}}{4}\left\|u^{-}\right\|_{H_{V}^{1}}^{2}+\frac{\left(s^{2}-t^{2}\right)^{2}}{4} \int_{\mathbb{R}^{3}} \phi_{u^{+}}\left(u^{-}\right)^{2} d x .
\end{aligned}
$$

The proof is completed.

Corollary 3.2 Suppose that $(V),\left(f_{1}\right),\left(f_{2}\right)$, and $\left(f_{4}\right)$ are satisfied. If $u=u^{+}+u^{-} \in \mathcal{M}_{\lambda}$, then

$$
J_{\lambda}\left(u^{+}+u^{-}\right)=\max _{s, t \geq 0} J_{\lambda}\left(s u^{+}+t u^{-}\right)
$$


Lemma 3.3 Assume that $(V)$ and $\left(f_{1}\right)-\left(f_{4}\right)$ are satisfied. If $u \in X$ with $u^{ \pm} \neq 0$, then there exists a unique pair $\left(s_{u}, t_{u}\right)$ of positive numbers such that $s_{u} u^{+}+t_{u} u^{-} \in \mathcal{M}_{\lambda}$.

Proof For each $u \in X$ with $u^{ \pm} \neq 0$, we first prove the existence by using the idea in [1]. Define the functions $g_{1}, g_{2}: \mathbb{R}_{+}^{2} \rightarrow \mathbb{R}$ by

$$
\begin{aligned}
g_{1}(s, t)= & \lambda s^{4}\left\|u^{+}\right\|_{W}^{4}+2 s^{4} \int_{\mathbb{R}^{3}}\left|\nabla u^{+}\right|^{2}\left(u^{+}\right)^{2} d x+s^{2}\left\|u^{+}\right\|_{H_{V}^{1}}^{2}+s^{4} \int_{\mathbb{R}^{3}} \phi_{u^{+}}\left(u^{+}\right)^{2} d x \\
& +s^{2} t^{2} \int_{\mathbb{R}^{3}} \phi_{u^{-}}\left(u^{+}\right)^{2} d x-\int_{\mathbb{R}^{3}} f\left(s u^{+}\right) s u^{+} d x
\end{aligned}
$$

and

$$
\begin{aligned}
g_{2}(s, t)= & \lambda t^{4}\left\|u^{-}\right\|_{W}^{4}+2 t^{4} \int_{\mathbb{R}^{3}}\left|\nabla u^{-}\right|^{2}\left(u^{-}\right)^{2} d x+t^{2}\left\|u^{-}\right\|_{H_{V}^{1}}^{2}+t^{4} \int_{\mathbb{R}^{3}} \phi_{u^{-}}\left(u^{-}\right)^{2} d x \\
& +s^{2} t^{2} \int_{\mathbb{R}^{3}} \phi_{u^{+}}\left(u^{-}\right)^{2} d x-\int_{\mathbb{R}^{3}} f\left(t u^{-}\right) t u^{-} d x .
\end{aligned}
$$

It is easy to see, by using $\left(f_{1}\right)-\left(f_{3}\right)$, that $g_{1}(s, s)>0$ and $g_{2}(s, s)>0$ for $s>0$ small enough and $g_{1}(t, t)<0, g_{2}(t, t)<0$ for $t>0$ large. Hence, there exist $0<a_{1}<a_{2}$ such that

$$
g_{1}\left(a_{1}, a_{1}\right)>0, \quad g_{2}\left(a_{1}, a_{1}\right)>0 ; \quad g_{1}\left(a_{2}, a_{2}\right)<0, \quad g_{2}\left(a_{2}, a_{2}\right)<0 .
$$

Notice that, for any fixed $s>0, g_{1}(s, t)$ is nondecreasing on $t \in[0, \infty)$ and, for any fixed $t>0, g_{2}(s, t)$ is nondecreasing on $s \in[0, \infty)$. Thereby, combining (3.1), (3.2) with (3.3), we have

$$
g_{1}\left(a_{1}, t\right)>0, \quad g_{1}\left(a_{2}, t\right)<0, \quad \forall t \in\left[a_{1}, a_{2}\right]
$$

and

$$
g_{2}\left(s, a_{1}\right)>0, \quad g_{2}\left(s, a_{2}\right)<0, \quad \forall s \in\left[a_{1}, a_{2}\right]
$$

By Miranda's theorem [26], there exists a pair $\left(s_{u}, t_{u}\right)$ with $a_{1}<s_{u}, t_{u}<a_{2}$ such that $g_{1}\left(s_{u}, t_{u}\right)=g_{2}\left(s_{u}, t_{u}\right)=0$. Hence, $s_{u} u^{+}+t_{u} u^{-} \in \mathcal{M}_{\lambda}$.

Next, we prove the uniqueness. Let $\left(s_{1}, t_{1}\right)$ and $\left(s_{2}, t_{2}\right)$ be such that $s_{i} u^{+}+t_{i} u^{-} \in \mathcal{M}_{\lambda}$, $i=1$, 2. Invoking Lemma 3.1, it yields

$$
\begin{aligned}
J_{\lambda}\left(s_{1} u^{+}+t_{1} u^{-}\right) \geq & J_{\lambda}\left(s_{2} u^{+}+t_{2} u^{-}\right)+\frac{\left(s_{1}^{2}-s_{2}^{2}\right)^{2}}{4 s_{1}^{2}}\left\|u^{+}\right\|_{H_{V}^{1}}^{2} \\
& +\frac{\left(t_{1}^{2}-t_{2}^{2}\right)^{2}}{4 t_{1}^{2}}\left\|u^{-}\right\|_{H_{V}^{1}}^{2},
\end{aligned}
$$

and

$$
J_{\lambda}\left(s_{2} u^{+}+t_{2} u^{-}\right) \geq J_{\lambda}\left(s_{1} u^{+}+t_{1} u^{-}\right)+\frac{\left(s_{1}^{2}-s_{2}^{2}\right)^{2}}{4 s_{2}^{2}}\left\|u^{+}\right\|_{H_{V}^{1}}^{2}+\frac{\left(t_{1}^{2}-t_{2}^{2}\right)^{2}}{4 t_{2}^{2}}\left\|u^{-}\right\|_{H_{V}^{1}}^{2} .
$$

This implies $\left(s_{1}, t_{1}\right)=\left(s_{2}, t_{2}\right)$. Thus we complete the proof. 
Lemma 3.4 Suppose that $(V)$ and $\left(f_{3}\right)$ hold. Forfixed $\lambda \in(0,1]$, let $m_{\lambda}=\inf _{u \in \mathcal{M}_{\lambda}} J_{\lambda}(u)$, then $m_{\lambda}>0$.

Proof For each $v \in \mathcal{M}_{\lambda},\left\langle J_{\lambda}^{\prime}(v), v\right\rangle=0$, then we claim that there exists a constant $a>0$ such that $\|v\|^{2}>a$ for each $v \in \mathcal{M}_{\lambda}$. In fact, we use an argument of contradiction and suppose that there exists a sequence $\left\{v_{n}\right\} \subset \mathcal{M}_{\lambda}$ such that $\left\|v_{n}\right\| \rightarrow 0$. Thus $a_{n}=\left\|v_{n}\right\|_{W} \rightarrow 0$ and $b_{n}=\left\|v_{n}\right\|_{H_{V}^{1}} \rightarrow 0$. According to conditions $\left(f_{1}\right)$ and $\left(f_{2}\right)$, we observe that, for any given $\varepsilon>0$, there exists $C_{\varepsilon}>0$ such that

$$
|f(s)| \leq \varepsilon|s|+C_{\varepsilon}|s|^{11}, \quad s \in \mathbb{R} .
$$

Then, by virtue of the Sobolev embedding theorem, we have

$$
\begin{gathered}
\lambda \int_{\mathbb{R}^{3}}\left(\left|\nabla v_{n}\right|^{4}+v_{n}^{4}\right) d x+\int_{\mathbb{R}^{3}}\left(\left|\nabla v_{n}\right|^{2}+V(x) v_{n}^{2}\right) d x \\
\quad+\int_{\mathbb{R}^{3}} \phi_{v_{n}} v_{n}^{2} d x+2 \int_{\mathbb{R}^{3}}\left|\nabla v_{n}\right|^{2} v_{n}^{2} d x \\
=\int_{\mathbb{R}^{3}} f\left(v_{n}\right) v_{n} d x \leq \varepsilon\left\|v_{n}\right\|_{2}^{2}+C\left\|v_{n}\right\|^{12} .
\end{gathered}
$$

Hence, for $b_{n}<1$, it follows

$$
\begin{aligned}
a_{n}^{4}+b_{n}^{4} & \leq a_{n}^{4}+b_{n}^{2} \leq C\left(a_{n}^{2}+b_{n}^{2}\right)^{6} \\
& \leq C_{1}\left(a_{n}^{12}+b_{n}^{12}\right) \leq C_{1}\left(a_{n}^{4}+b_{n}^{4}\right)^{3},
\end{aligned}
$$

which is a contradiction. For each $v \in \mathcal{M}_{\lambda},\left\langle J_{\lambda}^{\prime}(v), v\right\rangle=0$. Thus, we deduce, by $\left(f_{3}\right)$, that there exists a constant $\eta>0$ such that

$$
\begin{aligned}
J_{\lambda}(v)= & J_{\lambda}(v)-\frac{1}{\mu}\left\langle J_{\lambda}^{\prime}(v), v\right\rangle \\
= & \left(\frac{1}{4}-\frac{1}{\mu}\right) \lambda\|v\|_{W}^{4}+\left(\frac{1}{2}-\frac{1}{\mu}\right)\|v\|_{H_{V}^{1}}^{2}+\left(\frac{1}{2}-\frac{2}{\mu}\right) \int_{\mathbb{R}^{3}} v^{2}|\nabla v|^{2} d x \\
& +\left(\frac{1}{4}-\frac{1}{\mu}\right) \int_{\mathbb{R}^{3}} \phi_{v} v^{2} d x+\int_{\mathbb{R}^{3}}\left(\frac{1}{\mu} f(v) v-F(v)\right) \\
\geq & \left(\frac{1}{4}-\frac{1}{\mu}\right) \lambda\|v\|_{W}^{4}+\left(\frac{1}{2}-\frac{1}{\mu}\right)\|v\|_{H_{V}^{1}}^{2} \geq \eta,
\end{aligned}
$$

which implies that $m_{\lambda} \geq \eta>0$. The proof is completed.

Lemma 3.5 Suppose that $(V)$ and $\left(f_{1}\right)-\left(f_{4}\right)$ are satisfied. Then

$$
\inf _{u \in \mathcal{M}_{\lambda}} J_{\lambda}(u)=m_{\lambda}=\inf _{u \in H, u^{ \pm} \neq 0} \max _{s, t \geq 0} J_{\lambda}\left(s u^{+}+t u^{-}\right)
$$

Proof It follows from Corollary 3.2 that

$$
\inf _{u \in X, u^{ \pm} \neq 0} \max _{s, t \geq 0} J_{\lambda}\left(s u^{+}+t u^{-}\right) \leq \inf _{u \in \mathcal{M}_{\lambda}} \max _{s, t \geq 0} J_{\lambda}\left(s u^{+}+t u^{-}\right)=\inf _{u \in \mathcal{M}_{\lambda}} J_{\lambda}(u)=m_{\lambda}
$$


On the other hand, for any $u \in X$ with $u^{ \pm} \neq 0$, we deduce from Lemma 3.1 that

$$
\max _{s, t \geq 0} J_{\lambda}\left(s u^{+}+t u^{-}\right) \geq J_{\lambda}\left(s_{u} u^{+}+t_{u} u^{-}\right) \geq \inf _{u \in \mathcal{M}_{\lambda}} J_{\lambda}(u)=m_{\lambda}
$$

which ensures

$$
\inf _{u \in X, u^{ \pm} \neq 0} \max _{s, t \geq 0} J_{\lambda}\left(s u^{+}+t u^{-}\right) \geq \inf _{u \in \mathcal{M}_{\lambda}} J_{\lambda}(u)=m_{\lambda} .
$$

Thus, the conclusion holds. The proof is completed.

Lemma 3.6 Suppose that $(V)$ and $\left(f_{1}\right)-\left(f_{4}\right)$ are satisfied. For fixed $\lambda \in(0,1]$, the $m_{\lambda}$ can be achieved.

Proof Let $\left\{u_{n}\right\} \subset \mathcal{M}_{\lambda}$ be such that $J_{\lambda}\left(u_{n}\right) \rightarrow m_{\lambda}$. Then, for $n \in \mathbb{N}$ large enough, we obtain

$$
\begin{aligned}
m_{\lambda}+1 \geq & J_{\lambda}\left(u_{n}\right)-\frac{1}{\mu}\left\langle J_{\lambda}^{\prime}\left(u_{n}\right), u_{n}\right\rangle \\
= & \left(\frac{1}{4}-\frac{1}{\mu}\right) \lambda\left\|u_{n}\right\|_{W}^{4}+\left(\frac{1}{2}-\frac{1}{\mu}\right)\left\|u_{n}\right\|_{H_{V}^{1}}^{2}+\left(\frac{1}{2}-\frac{2}{\mu}\right) \int_{\mathbb{R}^{3}} u_{n}^{2}\left|\nabla u_{n}\right|^{2} d x \\
& +\left(\frac{1}{4}-\frac{1}{\mu}\right) \int_{\mathbb{R}^{3}} \phi_{u_{n}} u_{n}^{2} d x+\int_{\mathbb{R}^{3}}\left(\frac{1}{\mu} f\left(u_{n}\right) u_{n}-F\left(u_{n}\right)\right) .
\end{aligned}
$$

This shows that $\left\{u_{n}\right\}$ is bounded in $X$. Then, up to a subsequence, we assume that there exists $u_{\lambda} \in X$ such that $u_{n}^{ \pm} \rightarrow u_{\lambda}^{ \pm}$in $X$. Recall that the embedding from $H_{V}^{1}\left(\mathbb{R}^{3}\right)$ into $L^{2}\left(\mathbb{R}^{3}\right)$ is compact. Thus, by applying the interpolation inequality, it is easy to see that $u_{n} \rightarrow u$ in $L^{q}\left(\mathbb{R}^{3}\right)$ for $2 \leq q<12$. Since $u_{n} \in \mathcal{M}_{\lambda}$, there holds \langle\rangle$\left._{\lambda}^{\prime}\left(u_{n}\right), u_{n}^{ \pm}\right\rangle=0$, that is,

$$
\begin{aligned}
& \lambda \int_{\mathbb{R}^{3}}\left(\left|\nabla u_{n}^{ \pm}\right|^{4}+\left(u_{n}^{ \pm}\right)^{4}\right) d x+\int_{\mathbb{R}^{3}}\left(\left|\nabla u_{n}^{ \pm}\right|^{2}+V(x)\left(u_{n}^{ \pm}\right)^{2}\right) d x \\
& \quad+\int_{\mathbb{R}^{3}} \phi_{u_{n}}\left(u_{n}^{ \pm}\right)^{2} d x+2 \int_{\mathbb{R}^{3}}\left|\nabla u_{n}^{ \pm}\right|^{2}\left(u_{n}^{ \pm}\right)^{2} d x=\int_{\mathbb{R}^{3}} f\left(u_{n}^{ \pm}\right) u_{n}^{ \pm} d x .
\end{aligned}
$$

Repeating the above arguments once more, there exists a constant $\mu>0$ such that $\left\|u_{n}^{ \pm}\right\|^{2}>\mu$. It is easy to verify from $\left(f_{1}\right)$ and $\left(f_{2}\right)$ that, for any $\varepsilon>0$ and $p \in(2,12)$, there exists $C_{\varepsilon}>0$ such that

$$
|f(s)| \leq \varepsilon\left(|s|+|s|^{11}\right)+C_{\varepsilon}|s|^{p-1}, \quad s \in \mathbb{R} .
$$

Hence, we derive that, for some constant $\mu_{1}>0$,

$$
\begin{aligned}
0< & \mu_{1} \leq \liminf _{n \rightarrow \infty}\left[\lambda \int_{\mathbb{R}^{3}}\left(\left|\nabla u_{n}^{ \pm}\right|^{4}+\left(u_{n}^{ \pm}\right)^{4}\right) d x+\int_{\mathbb{R}^{3}}\left(\left|\nabla u_{n}^{ \pm}\right|^{2}+V(x)\left(u_{n}^{ \pm}\right)^{2}\right) d x\right. \\
& \left.+\int_{\mathbb{R}^{3}} \phi_{u_{n}}\left(u_{n}^{ \pm}\right)^{2} d x+2 \int_{\mathbb{R}^{3}}\left|\nabla u_{n}^{ \pm}\right|^{2}\left(u_{n}^{ \pm}\right)^{2} d x\right] \\
= & \liminf _{n \rightarrow \infty} \int_{\mathbb{R}^{3}} f\left(u_{n}^{ \pm}\right) u_{n}^{ \pm} d x \\
= & \int_{\mathbb{R}^{3}} f\left(u_{\lambda}^{ \pm}\right) u_{\lambda}^{ \pm} d x,
\end{aligned}
$$


which implies that $u_{\lambda}^{ \pm} \neq 0$. It follows from Lemma 3.2 that there exists a unique pair of positive numbers $\left(s_{1}, t_{1}\right)$ such that $s_{1} u_{\lambda}^{+}+t_{1} u_{\lambda}^{-} \in \mathcal{M}_{\lambda}$. Thus, we have

$$
\begin{aligned}
& \lambda s_{1}^{4}\left\|u_{\lambda}^{+}\right\|_{W}^{4}+2 s_{1}^{4} \int_{\mathbb{R}^{3}}\left|\nabla u_{\lambda}^{+}\right|^{2}\left(u_{\lambda}^{+}\right)^{2} d x+s_{1}^{2}\left\|u_{\lambda}^{+}\right\|_{H_{V}^{1}}^{2}+s_{1}^{4} \int_{\mathbb{R}^{3}} \phi_{u_{\lambda}^{+}}\left(u_{\lambda}^{+}\right)^{2} d x \\
& \quad+s_{1}^{2} t_{1}^{2} \int_{\mathbb{R}^{3}} \phi_{u_{\lambda}^{-}}\left(u_{\lambda}^{+}\right)^{2} d x=\int_{\mathbb{R}^{3}} f\left(s_{1} u_{\lambda}^{+}\right) s_{1} u_{\lambda}^{+} d x .
\end{aligned}
$$

Without loss of the generality, we assume that $t_{1} \leq s_{1}$. Then, by using (3.1) again, we have

$$
\begin{aligned}
& \frac{1}{s_{1}^{2}}\left\|u_{\lambda}^{+}\right\|_{H_{V}^{1}}^{2}+\lambda\left\|u_{\lambda}^{+}\right\|_{W}^{4}+2 \int_{\mathbb{R}^{3}}\left|\nabla u_{\lambda}^{+}\right|^{2}\left(u_{\lambda}^{+}\right)^{2} d x+\int_{\mathbb{R}^{3}} \phi_{u_{\lambda}^{+}}\left(u_{\lambda}^{+}\right)^{2} d x \\
& \quad+\int_{\mathbb{R}^{3}} \phi_{u_{\lambda}^{-}}\left(u_{\lambda}^{+}\right)^{2} d x \geq \int_{\mathbb{R}^{3}} \frac{f\left(s_{1} u_{\lambda}^{+}\right)}{\left(s_{1} u_{\lambda}^{+}\right)^{3}}\left(u_{\lambda}^{+}\right)^{4} d x .
\end{aligned}
$$

According to the weak semicontinuity of norm, Fatou's lemma, and (3.4), we see that

$$
\begin{aligned}
\lambda \int_{\mathbb{R}^{3}} & \left(\left|\nabla u_{\lambda}^{ \pm}\right|^{4}+\left(u_{\lambda}^{ \pm}\right)^{4}\right) d x+\int_{\mathbb{R}^{3}}\left(\left|\nabla u_{\lambda}^{ \pm}\right|^{2}+V(x)\left(u_{\lambda}^{ \pm}\right)^{2}\right) d x \\
& +\int_{\mathbb{R}^{3}} \phi_{u_{\lambda}}\left(u_{\lambda}^{ \pm}\right)^{2} d x+2 \int_{\mathbb{R}^{3}}\left|\nabla u_{\lambda}^{ \pm}\right|^{2}\left(u_{\lambda}^{ \pm}\right)^{2} d x \\
\leq & \liminf _{n \rightarrow \infty}\left[\lambda \int_{\mathbb{R}^{3}}\left(\left|\nabla u_{n}^{ \pm}\right|^{4}+\left(u_{n}^{ \pm}\right)^{4}\right) d x+\int_{\mathbb{R}^{3}}\left(\left|\nabla u_{n}^{ \pm}\right|^{2}+V(x)\left(u_{n}^{ \pm}\right)^{2}\right) d x\right. \\
& \left.+\int_{\mathbb{R}^{3}} \phi_{u_{n}}\left(u_{n}^{ \pm}\right)^{2} d x+2 \int_{\mathbb{R}^{3}}\left|\nabla u_{n}^{ \pm}\right|^{2}\left(u_{n}^{ \pm}\right)^{2} d x\right] \\
= & \liminf _{n \rightarrow \infty} \int_{\mathbb{R}^{3}} f\left(u_{n}^{ \pm}\right) u_{n}^{ \pm} d x=\int_{\mathbb{R}^{3}} f\left(u_{\lambda}^{ \pm}\right) u_{\lambda}^{ \pm} d x,
\end{aligned}
$$

which shows that

$$
\left\langle J_{\lambda}^{\prime}\left(u_{\lambda}\right), u_{\lambda}^{ \pm}\right\rangle \leq 0 .
$$

If $s_{1}>1$, in view of (3.6) and $\left(f_{4}\right)$, we derive

$$
\begin{aligned}
& \left\|u_{\lambda}^{+}\right\|_{H_{V}^{1}}^{2}+\lambda\left\|u_{\lambda}^{+}\right\|_{W}^{4}+2 \int_{\mathbb{R}^{3}}\left|\nabla u_{\lambda}^{+}\right|^{2}\left(u_{\lambda}^{+}\right)^{2} d x+\int_{\mathbb{R}^{3}} \phi_{u_{\lambda}^{+}}\left(u_{\lambda}^{+}\right)^{2} d x \\
& \quad+\int_{\mathbb{R}^{3}} \phi_{u_{\lambda}^{-}}\left(u_{\lambda}^{+}\right)^{2} d x>\int_{\mathbb{R}^{3}} f\left(u_{\lambda}^{+}\right) u_{\lambda}^{ \pm} d x
\end{aligned}
$$

which contradicts that $\left\langle J_{\lambda}^{\prime}\left(u_{\lambda}\right), u_{\lambda}^{+}\right\rangle \leq 0$. Thus we conclude that $s_{1} \leq 1$. It follows from $\left(f_{4}\right)$ that $f(s) s / 4-F(s)$ is nondecreasing on $(0, \infty)$ and nonincreasing on $(-\infty, 0)$. Hence, by the definition of $m_{\lambda}$, we have

$$
\begin{aligned}
m_{\lambda} & =\lim _{n \rightarrow \infty}\left[J_{\lambda}\left(u_{n}\right)-\frac{1}{4}\left\langle J_{\lambda}^{\prime}\left(u_{n}\right), u_{n}\right\rangle\right] \\
& \geq \frac{1}{4} \liminf _{n \rightarrow \infty}\left\|u_{n}\right\|_{H_{V}^{1}}^{2}+\liminf _{n \rightarrow \infty} \int_{\mathbb{R}^{3}}\left(\frac{1}{4} f\left(u_{n}\right) u_{n}-F\left(u_{n}\right)\right) \\
& \geq \frac{1}{4}\left\|u_{\lambda}\right\|_{H_{V}^{1}}^{2}+\int_{\mathbb{R}^{3}}\left(\frac{1}{4} f\left(u_{\lambda}\right) u_{\lambda}-F\left(u_{\lambda}\right)\right)
\end{aligned}
$$




$$
\begin{aligned}
= & J_{\lambda}\left(u_{\lambda}\right)-\frac{1}{4}\left\langle J_{\lambda}^{\prime}\left(u_{\lambda}\right), u_{\lambda}\right\rangle \\
\geq & \frac{1}{4}\left[s_{1}^{2}\left\|u_{\lambda}^{+}\right\|_{H_{V}^{1}}^{2}+t_{1}^{2}\left\|u_{\lambda}^{-}\right\|_{H_{V}^{1}}^{2}\right] \\
& +\int_{\mathbb{R}^{3}}\left(\frac{1}{4} f\left(s_{1} u_{\lambda}^{+}\right) s_{1} u_{\lambda}^{+}-F\left(s_{1} u_{\lambda}^{+}\right)\right)+\int_{\mathbb{R}^{3}}\left(\frac{1}{4} f\left(t_{1} u_{\lambda}^{-}\right) t_{1} u_{\lambda}^{-}-F\left(t_{1} u_{\lambda}^{-}\right)\right) \\
= & J_{\lambda}\left(s_{1} u_{\lambda}^{+}+t_{1} u_{\lambda}^{-}\right)-\frac{1}{4}\left\langle J_{\lambda}^{\prime}\left(s_{1} u_{\lambda}^{+}+t_{1} u_{\lambda}^{-}\right), s_{1} u_{\lambda}^{+}+t_{1} u_{\lambda}^{-}\right\rangle \geq m_{\lambda} .
\end{aligned}
$$

Therefore, we observe that $s_{1}=t_{1}=1, u_{\lambda} \in \mathcal{M}_{\lambda}$, and $J_{\lambda}\left(u_{\lambda}\right)=m_{\lambda}$. The proof is completed.

Lemma 3.7 Assume that $(V)$ and $\left(f_{1}\right)-\left(f_{4}\right)$ are satisfied. If $v_{\lambda} \in \mathcal{M}_{\lambda}$ and $J_{\lambda}\left(v_{\lambda}\right)=m_{\lambda}$ for fixing $\lambda \in(0,1]$, then $v_{\lambda}$ is a critical point of $J_{\lambda}$.

Proof It is sufficient to prove that $J_{\lambda}^{\prime}\left(v_{\lambda}\right)=0$. Otherwise, there exist $\delta, \mu>0$ such that $\left\|J_{\lambda}^{\prime}(w)\right\| \geq \mu$ for all $w \in \bar{B}_{3 \delta}\left(v_{\lambda}\right)=\left\{w \in X:\left\|w-v_{\lambda}\right\| \leq 3 \delta\right\}$. Let $D=(0.5,1.5) \times(0.5,1.5)$ and define $\gamma(s, t)=s v_{\lambda}^{+}+t v_{\lambda}^{-}$on $\bar{D}$. From Corollary 3.2, we can derive that

$$
\kappa=\max _{(s, t) \in \partial D} J_{\lambda}(\gamma(s, t))<m_{\lambda}
$$

For $\varepsilon \in\left(0, \min \left\{\left(m_{\lambda}-\kappa\right) / 2, \mu \delta / 8\right\}\right)$ and $S=B_{\delta}\left(v_{\lambda}\right)=\left\{w \in X:\left\|w-v_{\lambda}\right\|<\delta\right\}$, there exists a deformation $\eta \in C([0,1] \times X, X)$ such that

(a) $\eta(1, w)=w, w \notin J_{\lambda}^{-1}\left(\left[m_{\lambda}-2 \varepsilon, m_{\lambda}+2 \varepsilon\right]\right) \cap S_{2 \delta}$;

(b) $\eta\left(1, J_{\lambda}^{m_{\lambda}+\varepsilon} \cap S\right) \subset J_{\lambda}^{m_{\lambda}-\varepsilon}$;

(c) $J_{\lambda}\left(\eta(1, w) \leq J_{\lambda}(w), w \in X\right.$;

(d) $\|\eta(1, w)-w\| \leq \delta, w \in X$.

It follows from Corollary 3.2 that $J_{\lambda}\left(s v_{\lambda}^{+}+t v_{\lambda}^{-}\right) \leq J_{\lambda}\left(v_{\lambda}\right)=m_{\lambda}$ for $s, t \geq 0$. By (b), it is easy to see that

$$
J_{\lambda}\left(\eta\left(1, s v_{\lambda}^{+}+t v_{\lambda}^{-}\right)\right) \leq m_{\lambda}-\varepsilon, \quad \forall s, t \geq 0, \quad|s-1|^{2}+|t-1|^{2}<\delta^{2} /\left\|v_{\lambda}\right\|^{2} .
$$

On the other hand, for $s, t \geq 0,|s-1|^{2}+|t-1|^{2} \geq \delta^{2} /\left\|v_{\lambda}\right\|^{2}$, we deduce, by (c) and Lemma 3.1, that

$$
\begin{aligned}
J_{\lambda}\left(\eta\left(1, s v_{\lambda}^{+}+t v_{\lambda}^{-}\right)\right) & \leq J_{\lambda}\left(s v_{\lambda}^{+}+t v_{\lambda}^{-}\right) \\
& \leq J_{\lambda}\left(v_{\lambda}\right)-\frac{\left(1-s^{2}\right)^{2}}{4}\left\|v_{\lambda}^{+}\right\|_{H_{V}^{1}}^{2}-\frac{\left(1-t^{2}\right)^{2}}{4}\left\|v_{\lambda}^{-}\right\|_{H_{V}^{1}}^{2} \\
& \leq m_{\lambda}-\frac{\delta^{2}}{8\left\|v_{\lambda}\right\|^{2}} \min \left\{\left\|v_{\lambda}^{+}\right\|_{H_{V}^{1}}^{2},\left\|v_{\lambda}^{-}\right\|_{H_{V}^{1}}^{2}\right\} .
\end{aligned}
$$

Hence, combining (3.7) with (3.8), we have

$$
\max _{(s, t) \in \bar{D}} J_{\lambda}\left(\eta\left(1, s v_{\lambda}^{+}+t v_{\lambda}^{-}\right)\right)<m_{\lambda}
$$


In what follows, we prove that $\eta(1, \gamma(D)) \cap \mathcal{M}_{\lambda} \neq \emptyset$. Let us define functions on $\bar{D}$ by

$$
\begin{aligned}
& \gamma_{1}(s, t)=\eta(1, \gamma(s, t)), \\
& \Psi_{0}(s, t)=\left(\left\langle J_{\lambda}^{\prime}(\gamma(s, t)), v_{\lambda}^{+}\right\rangle,\left\langle J_{\lambda}^{\prime}(\gamma(s, t)), v_{\lambda}^{-}\right\rangle\right),
\end{aligned}
$$

and

$$
\Psi_{1}(s, t)=\left(\frac{1}{s}\left\langle J_{\lambda}^{\prime}\left(\gamma_{1}(s, t)\right),\left(\gamma_{1}(s, t)\right)^{+}\right\rangle, \frac{1}{t}\left\langle J_{\lambda}^{\prime}\left(\gamma_{1}(s, t)\right),\left(\gamma_{1}(s, t)\right)^{-}\right\rangle\right) .
$$

By direct calculation, we derive that the Jacobi matrix of $\Psi_{0}$ at $(1,1)$ is

$$
J_{\Psi_{0}}(1,1)=\left(\begin{array}{cc}
A & 2 \int_{\mathbb{R}^{3}} \phi_{v_{\lambda}^{-}}\left(v_{\lambda}^{+}\right)^{2} d x \\
2 \int_{\mathbb{R}^{3}} \phi_{v_{\lambda}^{+}}\left(v_{\lambda}^{-}\right)^{2} d x & B
\end{array}\right),
$$

where

$$
\begin{aligned}
A= & 3 \lambda\left\|v_{\lambda}^{+}\right\|_{W}^{4}+6 \int_{\mathbb{R}^{3}}\left|\nabla v_{\lambda}^{+}\right|^{2}\left(v_{\lambda}^{+}\right)^{2} d x+\left\|v_{\lambda}^{+}\right\|_{H_{V}^{1}}^{2}+3 \int_{\mathbb{R}^{3}} \phi_{v_{\lambda}^{+}}\left(v_{\lambda}^{+}\right)^{2} d x \\
& +\int_{\mathbb{R}^{3}} \phi_{v_{\lambda}^{-}}\left(v_{\lambda}^{+}\right)^{2} d x-\int_{\mathbb{R}^{3}} f^{\prime}\left(v_{\lambda}^{+}\right)\left(v_{\lambda}^{+}\right)^{2} d x,
\end{aligned}
$$

and

$$
\begin{aligned}
B= & 3 \lambda\left\|v_{\lambda}^{-}\right\|_{W}^{4}+6 \int_{\mathbb{R}^{3}}\left|\nabla v_{\lambda}^{-}\right|^{2}\left(v_{\lambda}^{-}\right)^{2} d x+\left\|v_{\lambda}^{-}\right\|_{H_{V}^{1}}^{2}+3 \int_{\mathbb{R}^{3}} \phi_{v_{\lambda}^{-}}\left(v_{\lambda}^{-}\right)^{2} d x \\
& +\int_{\mathbb{R}^{3}} \phi_{v_{\lambda}^{+}}\left(v_{\lambda}^{-}\right)^{2} d x-\int_{\mathbb{R}^{3}} f^{\prime}\left(v_{\lambda}^{-}\right)\left(v_{\lambda}^{-}\right)^{2} d x .
\end{aligned}
$$

We deduce from $\left(f_{4}\right)$, by simple calculation, that

$$
3 f(s) s \leq f^{\prime}(s) s^{2}, \quad s \in \mathbb{R} .
$$

Hence, recalling that $v_{\lambda} \in \mathcal{M}_{\lambda}$, we derive that

$$
\begin{aligned}
A & =-2\left(\left\|v_{\lambda}^{+}\right\|_{H_{V}^{1}}^{2}+\int_{\mathbb{R}^{3}} \phi_{v_{\lambda}^{-}}\left(v_{\lambda}^{+}\right)^{2} d x\right)+\int_{\mathbb{R}^{3}}\left[3 f\left(v_{\lambda}^{+}\right) v_{\lambda}^{+}-f^{\prime}\left(v_{\lambda}^{+}\right)\left(v_{\lambda}^{+}\right)^{2}\right] d x \\
& \leq-2\left(\left\|v_{\lambda}^{+}\right\|_{H_{V}^{1}}^{2}+\int_{\mathbb{R}^{3}} \phi_{v_{\lambda}^{-}}\left(v_{\lambda}^{+}\right)^{2} d x\right) .
\end{aligned}
$$

Similarly,

$$
B \leq-2\left(\left\|v_{\lambda}^{-}\right\|_{H_{V}^{1}}^{2}+\int_{\mathbb{R}^{3}} \phi_{\nu_{\lambda}^{+}}\left(v_{\lambda}^{-}\right)^{2} d x\right) .
$$

Hence, we can conclude that

$$
\operatorname{det} J_{\Psi_{0}}(1,1) \geq 4\left\|v_{\lambda}^{+}\right\|_{H_{V}^{1}}^{2}\left\|v_{\lambda}^{-}\right\|_{H_{V}^{1}}^{2}>0
$$


By using the fact that $(1,1)$ is the unique zero point of $\Psi_{0}$ in $\bar{D}$, we see that

$$
\operatorname{deg}\left(\Psi_{0}, D, 0\right)=\operatorname{sign} \operatorname{det} J_{\Psi_{0}}(1,1)=1
$$

Moreover, it follows from $(a)$ that $\gamma_{1}=\gamma$ on $\partial D$. Thus, we derive that $\operatorname{deg}\left(\Psi_{1}, D, 0\right)=$ $\operatorname{deg}\left(\Psi_{0}, D, 0\right)=1$. Therefore, there exists some $\left(s_{0}, t_{0}\right) \in D$ such that $\Psi_{1}\left(s_{0}, t_{0}\right)=0$. Then it follows from the Sobolev embedding theorem and (d) that $\gamma_{1}\left(s_{0}, t_{0}\right) \in \mathcal{M}_{\lambda}$ and hence $\eta(1, \gamma(D)) \cap \mathcal{M}_{\lambda} \notin \emptyset$, which is in contradiction with (3.9). Thereby, $u_{\lambda}$ is a sign-changing critical point of $J_{\lambda}$.

To the end, we will show that $u_{\lambda}$ has exactly two nodal domains, we assume by contradiction that

$$
u_{\lambda}=u_{1}+u_{2}+u_{3}
$$

where

$$
u_{i} \neq 0, \quad u_{1} \geq 0, \quad u_{2} \leq 0 \quad \text { and } \quad \operatorname{supp}\left(u_{i}\right) \cap \operatorname{supp}\left(u_{j}\right)=\emptyset, \quad \text { for } i \neq j, i, j=1,2,3 .
$$

It is obvious that

$$
\left\langle J_{\lambda}^{\prime}\left(u_{\lambda}\right), u_{i}\right\rangle=0 \quad \text { for } i=1,2,3 \text {. }
$$

Let $v=u_{1}+u_{2}$, we see that $v^{+}=u_{1}$ and $v^{-}=u_{2}$, and thus $v^{ \pm} \neq 0$. Then, it follows from $\left(f_{4}\right)$ and Lemma 3.1 that

$$
\begin{aligned}
m_{\lambda}= & J_{\lambda}\left(u_{\lambda}\right)=J_{\lambda}\left(u_{\lambda}\right)-\frac{1}{4}\left\langle J_{\lambda}^{\prime}\left(u_{\lambda}\right), u_{\lambda}\right\rangle \\
= & J_{\lambda}(v)+J_{\lambda}\left(u_{3}\right)+\frac{1}{4} \int\left(\phi_{u_{3}} v^{2}+\phi_{\nu} u_{3}^{2}\right) d x \\
& -\frac{1}{4}\left[\left\langle J_{\lambda}^{\prime}(v), v\right\rangle+\left\langle J_{\lambda}^{\prime}\left(u_{3}\right), u_{3}\right\rangle+\int_{\mathbb{R}^{3}}\left(\phi_{u_{3}} v^{2}+\phi_{\nu} u_{3}^{2}\right) d x\right] \\
\geq & \sup _{s, t \geq 0}\left[J_{\lambda}\left(s v^{+}+t v^{-}\right)+\frac{1-s^{4}}{4}\left\langle J_{\lambda}^{\prime}(v), v^{+}\right\rangle+\frac{1-t^{4}}{4}\left\langle J_{\lambda}^{\prime}(v), v^{-}\right\rangle\right] \\
& -\frac{1}{4}\left\langle J_{\lambda}^{\prime}(v), v\right\rangle+J_{\lambda}\left(u_{3}\right)-\frac{1}{4}\left\langle J_{\lambda}^{\prime}\left(u_{3}\right), u_{3}\right\rangle \\
\geq & \sup _{s, t \geq 0}\left[J_{\lambda}\left(s v^{+}+t v^{-}\right)+\frac{s^{4}}{4} \int \phi_{u_{3}}\left(v^{+}\right)^{2} d x+\frac{t^{4}}{4} \int_{\mathbb{R}^{3}} \phi_{u_{3}}\left(v^{-}\right)^{2} d x\right] \\
& +\frac{1}{4}\left\|u_{3}\right\|_{H_{V}^{1}}^{2}+\int_{\mathbb{R}^{3}}\left[\frac{1}{4} f\left(u_{3}\right) u_{3}-F\left(u_{3}\right)\right] d x \\
\geq & \sup _{s, t \geq 0} J_{\lambda}\left(s v^{+}+t v^{-}\right)+\frac{1}{4}\left\|u_{3}\right\|_{H_{V}^{1}}^{2} \\
\geq & m_{\lambda}+\frac{1}{4}\left\|u_{3}\right\|_{H_{V}^{1}}^{2},
\end{aligned}
$$

which implies that $u_{3}=0$, and $u_{\lambda}$ has exactly two nodal domains. The proof is completed. 
Lemma 3.8 ([16]) Let $\lambda_{n} \rightarrow 0$ as $n \rightarrow \infty$ and $\left\{u_{n}\right\} \subset X$ be a sequence of critical points of $J_{\lambda_{n}}$ satisfying $J_{\lambda_{n}}^{\prime}\left(u_{n}\right)=0$ and $J_{\lambda_{n}}\left(u_{n}\right) \leq C$ for some $C$ independent of $n$. Then up to a subsequence $u_{n} \rightarrow u$ in $H_{V}^{1}\left(\mathbb{R}^{3}\right)$ as $n \rightarrow \infty$ and $u$ is a critical point of $J$.

Proof of Theorem 1.1 Let us choose a sequence $\lambda_{n} \rightarrow 0$. By Lemma 3.6, there exists $\left\{u_{n}\right\} \subset$ $X$ satisfying $J_{\lambda_{n}}\left(u_{n}\right)=m_{\lambda_{n}}$ and $J_{\lambda_{n}}^{\prime}\left(u_{n}\right)=0$. Assume $\varphi \in C_{0}^{\infty}\left(\mathbb{R}^{3}\right)$ with $\varphi^{ \pm} \neq 0$, we can find a pair of positive numbers $\left(s_{0}, t_{0}\right)$ independent of $n$ such that

$$
\left\langle J_{\lambda_{n}}^{\prime}\left(s_{0} \varphi^{+}+t_{0} \varphi^{-}\right), s_{0} \varphi^{+}\right\rangle \leq\left\langle J_{1}^{\prime}\left(s_{0} \varphi^{+}+t_{0} \varphi^{-}\right), s_{0} \varphi^{+}\right\rangle<0
$$

and

$$
\left\langle J_{\lambda_{n}}^{\prime}\left(s_{0} \varphi^{+}+t_{0} \varphi^{-}\right), t_{0} \varphi^{-}\right\rangle \leq\left\langle J_{1}^{\prime}\left(s_{0} \varphi^{+}+t_{0} \varphi^{-}\right), t_{0} \varphi^{-}\right\rangle<0 .
$$

Let $\varphi_{1}=s_{0} \varphi^{+}+t_{0} \varphi^{-}$. Then similarly to the proof of Lemma 3.5, it follows from Lemma 3.1 that there exists a unique pair of positive numbers $\left(s_{n}, t_{n}\right) \subset(0,1] \times(0,1]$ such that $s_{n} \varphi_{1}^{+}+$ $t_{n} \varphi_{1}^{-} \in \mathcal{M}_{\lambda_{n}}$. Hence, by using $\left(f_{4}\right)$ again, we derive that

$$
\begin{aligned}
m_{\lambda_{n}} \leq & J_{\lambda_{n}}\left(s_{n} \varphi_{1}^{+}+t_{n} \varphi_{1}^{-}\right)-\frac{1}{4}\left\langle J_{\lambda_{n}}^{\prime}\left(s_{n} \varphi_{1}^{+}+t_{n} \varphi_{1}^{-}\right), s_{n} \varphi_{1}^{+}+t_{n} \varphi_{1}^{-}\right\rangle \\
= & \frac{1}{4}\left[s_{n}^{2}\left\|\varphi_{1}^{+}\right\|_{H_{V}^{1}}^{2}+t_{n}^{2}\left\|\varphi_{1}^{-}\right\|_{H_{V}^{1}}^{2}\right] \\
& +\int_{\mathbb{R}^{3}}\left(\frac{1}{4} f\left(s_{n} \varphi_{1}^{+}\right) s_{n} \varphi_{1}^{+}-F\left(s_{n} \varphi_{1}^{+}\right)\right)+\int_{\mathbb{R}^{3}}\left(\frac{1}{4} f\left(t_{n} \varphi_{1}^{-}\right) t_{n} \varphi_{1}^{-}-F\left(t_{n} \varphi_{1}^{-}\right)\right) \\
\leq & \frac{1}{4}\left[\left\|\varphi_{1}^{+}\right\|_{H_{V}^{1}}^{2}+\left\|\varphi_{1}^{-}\right\|_{H_{V}^{1}}^{2}\right]+\int_{\mathbb{R}^{3}}\left(\frac{1}{4} f\left(\varphi_{1}^{+}\right) \varphi_{1}^{+}-F\left(\varphi_{1}^{+}\right)\right) \\
& +\int_{\mathbb{R}^{3}}\left(\frac{1}{4} f\left(\varphi_{1}^{-}\right) \varphi_{1}^{-}-F\left(\varphi_{1}^{-}\right)\right) \\
= & J_{1}\left(\varphi_{1}\right)-\frac{1}{4}\left\langle J_{1}^{\prime}\left(\varphi_{1}\right), \varphi_{1}\right\rangle,
\end{aligned}
$$

which implies that $\left\{m_{\lambda_{n}}\right\}$ is bounded. Hence, according to Lemma 3.7, there exists a critical point $u$ of $J$ such that $u_{n} \rightarrow u$ in $H_{V}^{1}\left(\mathbb{R}^{3}\right)$, and hence $u_{n}^{ \pm} \rightarrow u^{ \pm}$in $L^{2}\left(\mathbb{R}^{3}\right)$. Next, we will show that $u^{ \pm} \neq 0$. In fact, if $\left\|u^{ \pm}\right\|_{2} \geq 1$, the result holds. On the other hand, suppose that $\left\|u^{ \pm}\right\|_{2}<1$, it follows that $\left\|u_{n}^{ \pm}\right\|_{2}<1$ for $n$ large enough. By using $\left(f_{1}\right)$ and $\left(f_{2}\right)$ again, there holds, for any $\varepsilon>0$ and $q \in(4,12)$, that there exists $C_{\varepsilon}>0$ such that

$$
|f(s)| \leq \varepsilon\left(|s|+|s|^{11}\right)+C_{\varepsilon}|s|^{q-1}, \quad s \in \mathbb{R} .
$$

Hence by Sobolev's inequality, interpolation inequality, and Young's inequality, we obtain that there exist $C_{1}, C_{2}>0$ such that

$$
\begin{aligned}
0= & \lambda_{n} \int_{\mathbb{R}^{3}}\left(\left|\nabla u_{n}^{ \pm}\right|^{4}+\left(u_{n}^{ \pm}\right)^{4}\right) d x+\int_{\mathbb{R}^{3}}\left(\left|\nabla u_{n}^{ \pm}\right|^{2}+V(x)\left(u_{n}^{ \pm}\right)^{2}\right) d x \\
& +2 \int_{\mathbb{R}^{3}}\left|\nabla u_{n}^{ \pm}\right|^{2}\left(u_{n}^{ \pm}\right)^{2} d x \\
& +\int_{\mathbb{R}^{3}} \phi_{u_{n}}\left(u_{n}^{ \pm}\right)^{2} d x-\int_{\mathbb{R}^{3}} f\left(u_{n}^{ \pm}\right) u_{n}^{ \pm} d x
\end{aligned}
$$




$$
\begin{aligned}
\geq & \int_{\mathbb{R}^{3}}\left(\left|\nabla u_{n}^{ \pm}\right|^{2}+V(x)\left(u_{n}^{ \pm}\right)^{2}\right) d x+\int_{\mathbb{R}^{3}}\left|\nabla u_{n}^{ \pm}\right|^{2}\left(u_{n}^{ \pm}\right)^{2} d x-\varepsilon\left\|u_{n}^{ \pm}\right\|_{2}^{2} \\
& -\varepsilon\left\|u_{n}^{ \pm}\right\|_{12}^{12}-C_{\varepsilon}\left\|u_{n}^{ \pm}\right\|_{q}^{q} \\
\geq & C_{1}\left\|u_{n}^{ \pm}\right\|_{q}^{4}-C_{2}\left\|u_{n}^{ \pm}\right\|_{q}^{q}
\end{aligned}
$$

which implies $\left\|u_{n}^{ \pm}\right\|_{q} \geq\left(\frac{C_{1}}{C_{2}}\right)^{1 /(q-4)}$. Note that the embedding from $H_{V}^{1}\left(\mathbb{R}^{3}\right)$ into $L^{2}\left(\mathbb{R}^{3}\right)$ is compact. Thus, by applying the interpolation inequality, we know that $u_{n} \rightarrow u$ in $L^{q}\left(\mathbb{R}^{3}\right)$ for $2 \leq q<12$. Thereby, we see that $u^{ \pm} \neq 0$ and $u$ is a sign-changing critical of $J$.

In the sequel, we claim that $u$ has also exactly two nodal domains. In fact, recalling that $u$ is a critical point of $J$, there holds

$$
\int_{\mathbb{R}^{3}}\left(|\nabla u|^{2}+V(x) u^{2}\right) d x+2 \int_{\mathbb{R}^{3}} u^{2}|\nabla u|^{2} d x+\int_{\mathbb{R}^{3}} \phi_{u} u^{2} d x=\int_{\mathbb{R}^{3}} f(u) u d x .
$$

On the other hand, $\left\langle J^{\prime}\left(u_{n}\right), u_{n}\right\rangle=0$ implies that

$$
\begin{aligned}
& \lambda_{n} \int_{\mathbb{R}^{3}}\left(\left|\nabla u_{n}\right|^{4}+u_{n}^{4}\right) d x+\int_{\mathbb{R}^{3}}\left(\left|\nabla u_{n}\right|^{2}+V(x) u_{n}^{2}\right) d x+2 \int_{\mathbb{R}^{3}} u_{n}^{2}\left|\nabla u_{n}\right|^{2} d x \\
& \quad+\int_{\mathbb{R}^{3}} \phi_{u_{n}} u_{n}^{2} d x=\int_{\mathbb{R}^{3}} f\left(u_{n}\right) u_{n} d x .
\end{aligned}
$$

By using (3.5) again and the compact embedding, we can prove

$$
\lim _{n \rightarrow \infty} \int_{\mathbb{R}^{3}} f\left(u_{n}\right) u_{n} d x=\int_{\mathbb{R}^{3}} f(u) u d x .
$$

Then, combining (3.10) with (3.11) and using Fatou's lemma and weak semicontinuity of norm, up to a subsequence, we get that $u_{n} \rightarrow u$ strongly in $H_{V}^{1}\left(\mathbb{R}^{3}\right)$. The proof is completed.

\section{Acknowledgements}

The author greatly thanks Professor Fuyi Li for his great help and many valuable discussions and would like to express their sincere gratitude to one anonymous referee for his/her constructive comments for improving the quality of this paper.

\section{Funding}

The paper is supported by the National Natural Science Foundation of China (Grant Nos. 11571209, 11501318, 11671239) and the Natural Science Foundation of Shanxi Province (Grant Nos. 201801D211001, 201801D121002, 201801D221012).

\section{Availability of data and materials}

Not applicable.

\section{Competing interests}

The authors declare that they have no competing interests.

\section{Authors' contributions}

The authors declare that this study was independently finished. All authors read and approved the final manuscript.

\section{Author details}

${ }^{1}$ School of Applied Mathematics, Shanxi University of Finance and Economics, Taiyuan, People's Republic of China. ${ }^{2}$ School of Mathematical Sciences, Shanxi University, Taiyuan, People's Republic of China. ${ }^{3}$ School of Mathematical Sciences, Qufu Normal University, Qufu, People's Republic of China.

\section{Publisher's Note}

Springer Nature remains neutral with regard to jurisdictional claims in published maps and institutional affiliations. 


\section{References}

1. Alves, C., Souto, M.: Existence of least energy nodal solution for a Schrödinger-Poisson system in bounded domains. Z. Angew. Math. Phys. 65, 1153-1166 (2014)

2. Alves, C., Souto, M., Soares, S.: Schrödinger-Poisson equations without Ambrosetti-Rabinowitz condition. J. Math. Anal. Appl. 377, 584-592 (2011)

3. Alves, C., Wang, Y., Shen, Y.: Soliton solutions for a class of quasilinear Schrödinger equations with a parameter. J. Differ. Equ. 259, 318-343 (2015)

4. Ambrosetti, A., Ruiz, D.: Multiple bound states for the Schrödinger-Poisson problem. Commun. Contemp. Math. 10, 391-404 (2008)

5. Bartsch, T., Liu, Z.L., Weth, T.: Sign changing solutions of superlinear Schrödinger equations. Commun. Partial Differ. Equ. 29, 25-42 (2004)

6. Bartsch, T., Liu, Z.L., Weth, T.: Nodal solutions of a p-Laplacian equation. Proc. Lond. Math. Soc. 91, 129-152 (2005)

7. Bartsch, T., Wang, Z.Q.: Existence and multiple results for some superlinear elliptic problems on $\mathbb{R}^{N}$. Commun. Partial Differ. Equ. 20, 1725-1741 (1995)

8. Castro, A., Cossio, J., Neuberger, J.: A sign-changing solution for a superlinear Dirichlet problem. Rocky Mt. J. Math. 27 , 1041-1053 (1997)

9. Chen, J.H., Tang, X.H., Gao, Z., Cheng, B.T.: Ground state sign-changing solutions for a class of generalized quasilinear Schrödinger equations with a Kirchhoff-type perturbation. J. Fixed Point Theory Appl. 19, 3127-3149 (2017)

10. Chen, S.T., Tang, X.H.: Ground state sign-changing solutions for a class of Schrödinger-Poisson type problems in $\mathbb{R}^{3}$. Z. Angew. Math. Phys. 67, 102 (2016)

11. Colin, M., Jeanjean, L.: Solutions for a quasilinear Schrödinger equation: a dual approach. Nonlinear Anal. 56, 213-226 (2004)

12. Costa, D.G.: On a class of elliptic systems in $\mathbb{R}^{N}$. Electron. J. Differ. Equ. 1994, 7 (1994)

13. Deng, Y., Peng, S., Wang, J.: Nodal soliton solutions for quasilinear Schrödinger equations with critical exponent. J. Math. Phys. 54, 349-381 (2013)

14. Deng, Y., Peng, S., Yan, S.: Positive soliton solutions for generalized quasilinear Schrödinger equations with critical growth. J. Differ. Equ. 258, 115-147 (2015)

15. Deng, Y., Peng, S., Yan, S.: Critical exponents and solitary wave solutions for generalized quasilinear Schrödinger equations. J. Differ. Equ. 260, 1228-1262 (2016)

16. Feng, X., Zhang, Y.: Existence of non-trivial solution for a class of modified Schrödinger-Poisson equations via perturbation method. J. Math. Anal. Appl. 442, 673-684 (2016)

17. Figueiredo, G.M., Nascimento, R.G.: Existence of a nodal solution with minimal energy for a Kirchhoff equation. Math. Nachr. 288, 48-60 (2015)

18. Ghimenti, M., Van Schaftingen, J.: Nodal solutions for the Choquard equation. J. Funct. Anal. 271, 107-135 (2016)

19. Goubet, O., Hamraoui, E.: Blow-up of solutions to cubic nonlinear Schrödinger equations with defect: the radial case. Adv. Nonlinear Anal. 6(2), 183-197 (2017)

20. Jeanjean, L., Luo, T., Wang, Z.Q.: Multiple normalized solutions for quasi-linear Schrödinger equations. J. Differ. Equ. 259, 3894-3928 (2015)

21. Li, F., Zhu, X., Liang, Z:: Multiple solutions to a class of generalized quasilinear Schrödinger equations with a Kirchhoff-type perturbation. J. Math. Anal. Appl. 443, 11-38 (2016)

22. Liu, J.Q., Wang, Y.Q., Wang, Z.Q.: Soliton solutions for quasilinear Schrödinger equations II. J. Differ. Equ. 187, 473-493 (2003)

23. Liu, J.Q., Wang, Y.Q., Wang, Z.Q.: Solutions for quasilinear Schrödinger equations via the Nehari method. Commun Partial Differ. Equ. 29, 879-901 (2004)

24. Liu, J.Q., Wang, Z.Q.: Multiple solutions for quasilinear elliptic equations with a finite potential well. J. Differ. Equ. 257, 2874-2899 (2014)

25. Liu, X.Q., Liu, J.Q., Wang, Z.Q.: Quasilinear elliptic equations via perturbation method. Proc. Am. Math. Soc. 141 253-263 (2013)

26. Miranda, C: Unosservazione su un teorema di Brouwer. Boll. Unione Mat. Ital. 3, 5-7 (1940)

27. Noussair, E., Wei, J.: On the effect of the domain geometry on the existence and profile of nodal solution of some singularly perturbed semilinear Dirichlet problem. Indiana Univ. Math. J. 46, 1255-1271 (1997)

28. Omana, W., Willem, M.: Homoclinic orbits for a class of Hamiltonian systems. Differ. Integral Equ. 5, 1115-1120 (1992)

29. Poppenberg, M., Schmitt, K., Wang, Z.Q.: On the existence of soliton solutions to quasilinear Schrödinger equations. Calc. Var. Partial Differ. Equ. 14, 329-344 (2002)

30. Rabinowitz, P.H.: On a class of nonlinear Schrödinger equations. Z. Angew. Math. Phys. 43, $270-291$ (1992)

31. Ruiz, D.: The Schrödinger-Poisson equation under the effect of a nonlinear local term. J. Funct. Anal. 237, 655-674 (2006)

32. Ruiz, D., Siciliano, G.: Existence of ground states for a modified nonlinear Schrödinger equation. Nonlinearity 23 $1221-1233(2010)$

33. Shuai, W.: Sign-changing solutions for a class of Kirchhoff-type problem in bounded domains. J. Differ. Equ. 259 1256-1274 (2015)

34. Shuai, W., Wang, Q.: Existence and asymptotic behavior of sign-changing solutions for the nonlinear Schrödinger-Poisson system in $\mathbb{R}^{3}$. Z. Angew. Math. Phys. 66, 3267-3282 (2015)

35. Tang, X.H., Cheng, B.T.: Ground state sign-changing solutions for Kirchhoff type problems in bounded domains. J. Differ. Equ. 261, 2384-2402 (2016)

36. Trabelsi, S.: Well-posedness of a higher-order Schrödinger-Poisson-Slater system. Bound. Value Probl. 2018, 181 (2018)

37. Wang, L., Radulescu, V.D., Zhang, B.: Infinitely many solutions for fractional Kirchhoff-Schrödinger-Poisson systems. J. Math. Phys. 60, 011506 (2019)

38. Wang, Z.P., Zhou, H.S.: Positive solution for a nonlinear stationary Schrödinger-Poisson system in $\mathbb{R}^{3}$. Discrete Contin. Dyn. Syst. 18, 809-816 (2012)

39. Wang, Z.P. Zhou, H.S.: Sign-changing solutions for the nonlinear Schrödinger-Poisson system in $\mathbb{R}^{3}$. Calc. Var. Partial Differ. Equ. 52, 927-943 (2015) 
40. Wen, L., Chen, S.: Ground state solutions for asymptotically periodic Schrödinger-Poisson systems involving Hartree-type nonlinearities. Bound. Value Probl. 2018, 110 (2018)

41. Wu, K., Wu, X.: Infinitely many small energy solutions for a modified Kirchhoff-type equation in $\mathbb{R}^{N}$. Comput. Math Appl. 70, 592-602 (2015)

42. Wu, K., Wu, X.: Radial solutions for quasilinear Schrödinger equations without 4-superlinear condition. Appl. Math. Lett. 76, 53-59 (2018)

43. Wu, X., Wu, K.: Existence of positive solutions, negative solutions and high energy solutions for quasi-linear elliptic equations on $\mathbb{R}^{N}$. Nonlinear Anal., Real World Appl. 16, 48-64 (2014)

44. Zhang, J., Tang, X., Zhang, W.: Infinitely many solutions of quasilinear Schrödinger equation with sign-changing potential. J. Math. Anal. Appl. 420, 1762-1775 (2014)

45. Zhao, L., Liu, H., Zhao, F.: Existence and concentration of solutions for the Schrödinger-Poisson equations with steep well potential. J. Differ. Equ. 255, 1-23 (2013)

46. Zhao, L., Zhao, F.: On the existence of solutions for the Schrödinger-Poisson equations. J. Math. Anal. Appl. 346, 155-169 (2008)

Submit your manuscript to a SpringerOpen ${ }^{\circ}$ journal and benefit from:

- Convenient online submission

- Rigorous peer review

Open access: articles freely available online

- High visibility within the field

- Retaining the copyright to your article

Submit your next manuscript at $\boldsymbol{\nabla}$ springeropen.com 